\title{
Enzyme replacement with PEGylated cystathionine $\beta$-synthase ameliorates homocystinuria in murine model
}

\author{
Erez M. Bublil,, ${ }^{1}$ Tomas Majtan, ${ }^{1}$ Insun Park, ${ }^{1}$ Richard S. Carrillo, ${ }^{1}$ Helena Hůlková, ${ }^{2}$ Jakub Krijt, ${ }^{2}$ Viktor Kožich, ${ }^{2}$ and Jan P. Kraus \\ 'Department of Pediatrics, University of Colorado School of Medicine, Aurora, Colorado, USA. Innstitute of Inherited Metabolic Disorders, Charles University in Prague - First Faculty of Medicine, \\ Prague, Czech Republic.
}

\begin{abstract}
Homocystinuria, which typically results from cystathionine $\beta$-synthase (CBS) deficiency, is the most common defect of sulfur amino acid metabolism. CBS condenses homocysteine and serine to cystathionine that is then converted to cysteine. Individuals with homocystinuria have markedly elevated plasma levels of homocysteine and methionine and reduced concentrations of cystathionine and cysteine. Clinical disease manifestations include thromboembolism and neuropsychiatric, ocular, and skeletal complications. Here, we have shown that administration of PECylated CBS into the circulation of homocystinuria model mice alters the extra- and intracellular equilibrium of sulfur amino acids, resulting in a decrease of approximately $75 \%$ in plasma total homocysteine (tHcy) and normalization of cysteine concentrations. Moreover, the decrease in homocysteine and the normalization of cysteine in PEGylated CBS-treated model mice were accompanied by improvement of histopathological liver symptoms and increased survival. Together, these data suggest that CBS enzyme replacement therapy (ERT) is a promising approach for the treatment of homocystinuria and that ERT for metabolic diseases may not necessitate introduction of the deficient enzyme into its natural intracellular compartment.
\end{abstract}

\section{Introduction}

Classical homocystinuria (Online Mendelian Inheritance in Man [OMIM] \#236200) is recognized as the most common inborn error of sulfur amino acid metabolism. It was first described by Carson and Neill in 1962 (1), and the discovery of its underlying cause, namely a deficiency of the cystathionine $\beta$-synthase (CBS) enzyme, was described shortly thereafter (2).

CBS governs the unidirectional flow of sulfur from methionine to cysteine by operating at the intersection of the transmethylation, transsulfuration, and remethylation pathways. It catalyzes a $\beta$-replacement reaction in which serine condenses with homocysteine in a pyridoxal-5'-phosphate-dependent (PLP-dependent) manner to form cystathionine (3). Cystathionine can then be converted to cysteine by cystathionine $\gamma$-lyase (CGL). Thus, proper function of CBS is critical for the regulation of both cysteine and methionine metabolism (4), and accordingly, a compromised CBS activity, or lack thereof, leads to the biochemical and clinical manifestations of CBS-deficient homocystinuria (CBSDH).

Biochemically, CBSDH is characterized by highly elevated blood levels of homocysteine, methionine, and S-adenosyl-homocysteine (SAH), accompanied by low levels of cysteine and cystathionine. Some of the clinical manifestations of untreated homocystinuria include thromboembolism, connective tissue problems

Conflict of interest: This research was funded by Orphan Technologies Ltd. J.P. Kraus, T. Majtan, and E.M. Bublil are the inventors on a patent that is based on the results presented here (US patent no. 9,034,318)

Submitted: November 4, 2015; Accepted: March 22, 2016.

Reference information: / Clin Invest. 2016;126(6):2372-2384. doi:10.1172/JCI85396. such as dislocation of the ocular lens, Marfanoid habitus and osteoporosis, cognitive impairment, and other signs $(4,5)$.

Initial attempts to treat homocystinuria involved dietary restrictions to decrease methionine intake, thus avoiding the build-up of toxic homocysteine (6). It was later found that supplementation with the PLP cofactor precursor vitamin $\mathrm{B}_{6}$ relieves the clinical manifestations for approximately half of the patients $(7,8)$, although a complementary moderately protein-restricted diet may be necessary for these patients to achieve full metabolic control (9). The remaining half of the patients, the vitamin $B_{6}$ nonresponders, are subjected to a stringent methionine/proteinrestricted diet, but their compliance with this diet is poor (10). The diet is usually combined with a methionine-free and cysteineenriched amino acid mixture and often with betaine, which can serve as a methyl donor for the enzyme betaine-homocysteine methyltransferase to produce dimethylglycine and methionine from homocysteine (11). In addition, folate and vitamin $B_{12}$ supplement therapy promotes remethylation of homocysteine to methionine. This improves metabolic control by lowering homocysteine levels, in conjunction with the strict diet. In some countries, $\mathrm{CBSDH}$ is detected by screening newborns in the presymptomatic phase of the disease, which improves the outcome of these therapies (12).

An alternative treatment strategy for $\mathrm{CBSDH}$ that provides vitamin $\mathrm{B}_{6}$ nonresponders and partial responders with a therapy that improves their metabolic abnormalities, reduces the accumulation of toxic homocysteine in the circulation, and increases cystathionine and cysteine levels is highly desired. Such normalization of metabolite levels may delay the onset of CBSDH, prevent or reverse the symptoms, and allow this group of affected indi- 
viduals to enjoy an unrestricted or only mildly restricted diet and thus significantly improve their quality of life. To provide these benefits, a therapy must deal with the core deficiency underlying this condition, namely the aberrant CBS function. Accordingly, we envisioned that systemic introduction of CBS, in the form of enzyme replacement therapy (ERT), may be beneficial to homocystinuria patients.

ERT is well known for its successful application in lysosomal storage diseases (LSDs) and other genetic disorders. However, the use of ERT to treat CBSDH presents a number of specific obstacles that need to be addressed. First, in LSDs, for example, the exogenous enzyme is glycosylated and thus taken up by receptor binding to the sugar moiety, followed by uptake and trafficking into the lysosome. This natural uptake to the site of need is not a trait of CBS that can be exploited for homocystinuria. We asked whether introduction of a recombinant enzyme into the circulation, with no trafficking to its native intracellular environment, would favorably affect intracellular metabolism. We assumed that a significant decrease in extracellular homocysteine due to CBS administration can generate a concentration gradient, triggering a flux of homocysteine from intra- to extracellular spaces, where the administered enzyme can further process it, with the extracellular CBS thus serving as a homocysteine "sink."

A second obstacle is rooted within the structure and mode of activation of native human CBS (hCBS), which exists as a tetramer composed of 4 identical monomers. This form of the enzyme has a high tendency toward aggregation, which poses a major constraint on purification efforts (13). Additionally, CBS is allosterically regulated by S-adenosyl-methionine (SAM), which binds to the autoinhibitory $\mathrm{C}$-terminal regulatory domain and activates the enzyme by approximately 5 -fold in vitro (14). SAM levels in CBS-deficient patients and controls are $1.38 \mu \mathrm{M}$ and $0.093 \mu \mathrm{M}$, respectively (15), in both cases far below the $\mathrm{K}_{\text {act }}$ value for CBS $(17.7 \mu \mathrm{M})(16)$, which would prevent full activation of hCBS if administered to patients. However, it was previously determined that a human truncated CBS (htCBS), lacking 138 amino acids from the C-terminus (the regulatory domain), forms dimers and is constitutively activated, regardless of SAM. Moreover, this form of the enzyme tends to form fewer aggregates $(17,18)$. Together, these data imply that htCBS is better suited than the WT enzyme for use in the development of ERT to treat CBSDH.

In addition, key pharmacokinetic and pharmacodynamic considerations must be addressed. Therapeutic proteins, unlike small molecules, are exclusively delivered via parenteral routes, and treatment efficacy may be greatly impacted by their absorption, distribution, metabolism, and excretion (ADME) (19). Highmolecular-weight compounds, such as enzymes, have limited tissue penetration capability and are thus mainly present in the plasma. These proteins may then be maintained in the circulation for a short period of time, as they are removed from the bloodstream by several mechanisms (19). Ideally, administered CBS is expected to maintain high activity in plasma for a period of time that is sufficient to have a steady and significant effect on sulfur amino acid metabolism. Accordingly, enhancing ERT efficacy may require additional modifications to the protein in order to increase the retention time in vivo. This may be achieved by PEGylation, the addition of polyethyleneglycol (PEG) moieties onto the surface of the protein, a strategy that has become widely accepted. PEGylation was found to minimize proteolysis, immune response, and antigenicity, while increasing protein stability and size and reducing renal excretion (20).

Here, we present a body of work that characterizes several modified forms of htCBS and show for the first time to our knowledge that exogenously administered CBS, in which exposed cysteine residues are PEGylated, positively affects the aberrant concentration of sulfur metabolites in homocystinuric mice. Furthermore, our ERT restores metabolic control in the face of a standard diet, ameliorates histopathological changes in the liver, and improves survival of a KO mouse model. The delivery of PEGylated htCBS thus represents a potential new therapeutic approach for $\mathrm{CBSDH}$.

\section{Results}

Unmodified htCBS exhibits a short retention time in the circulation. The pharmacokinetic properties of a pharmacologically active substance that is administered into the circulation are greatly affected by natural mechanisms of ADME. Rapid clearance of the injected molecule may greatly impact treatment efficacy, and thus longer circulating half-lives are desired, which may translate into less frequent administrations or smaller doses that in turn minimize the side effects. To determine the pharmacokinetics of htCBS, a single dose of $5 \mathrm{mg} / \mathrm{kg}$ body weight (BW) was injected via the s.c., i.v., or i.p. route into C57BL/6J mice. As shown in Figure $1 \mathrm{~A}$, administration of htCBS via the i.v. or i.p. route reached the highest CBS-specific activity values during the first 4 hours after injection (observed peak plasma levels of up to 123 and $76 \mathrm{mU} / \mu \mathrm{l}$, respectively. See also Supplemental Table 1 for AUC values; supplemental material available online with this article; doi:10.1172/ JCI85396DS1). For the s.c. mode of delivery, lower specific activity values were recorded (highest value of $34 \mathrm{mU} / \mu \mathrm{l}$ plasma). All routes of administration demonstrated similar values at 8 hours after injection. Most important, however, is that 24 hours after injection, only residual htCBS activity was detected, regardless of the route used. The pharmacokinetic parameters (Supplemental Table 1) showed that the half-life for htCBS in circulation was 2.7 hours, suggesting that after roughly 6 half-lives, less than 20 hours, htCBS levels should be undetectable. The bioavailability of htCBS was $50 \%$ for the s.c. route, suggesting that s.c. delivery may be a clinical option for this therapeutic approach. The longer apparent htCBS half-lives for s.c. and i.p. delivery (Supplemental Table 1) can be explained by a slow absorption phase that occurs during the elimination phase, thereby extending the mean residency time and apparent half-life. However, for our purpose, a much longer half-life was desirable, and thus we moved on to test PEGylated species of htCBS.

The rapid decline in htCBS activity in vivo may be attributed to a facilitated clearance from the circulation and may also be attributed to the loss of enzymatic activity of htCBS once introduced into the circulation. To test the latter, we incubated the htCBS enzyme in vitro in mouse plasma at $37^{\circ} \mathrm{C}$ for up to 96 hours and measured activity every 24 hours. As shown in Figure 1B, no significant loss of activity was recorded for up to 72 hours of incubation. A modest $25 \%$ loss of activity was recorded after 96 hours of incubation. Thus, we conclude that clearance from the circulation, rather than loss of activity in plasma, accounts for the rapid loss of htCBS activity in the circulation, as also shown below. 


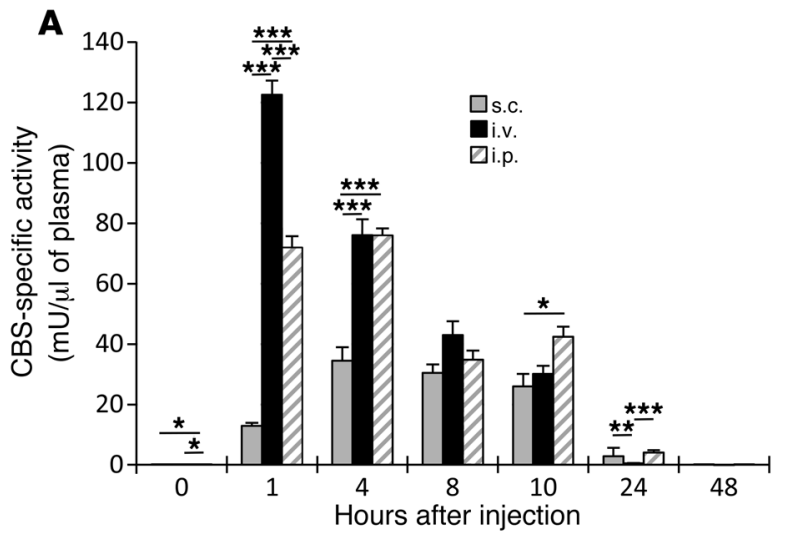

C

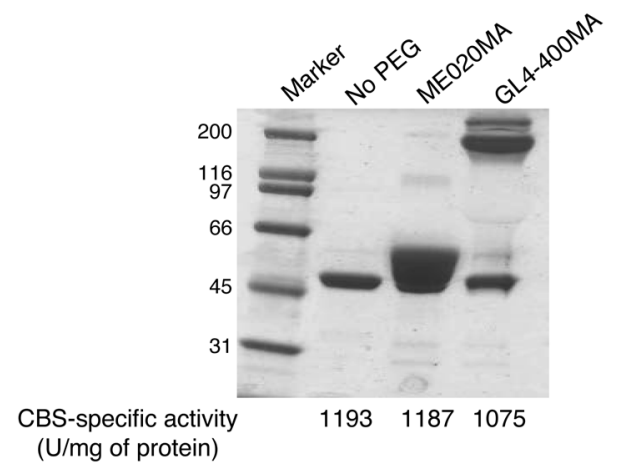

B
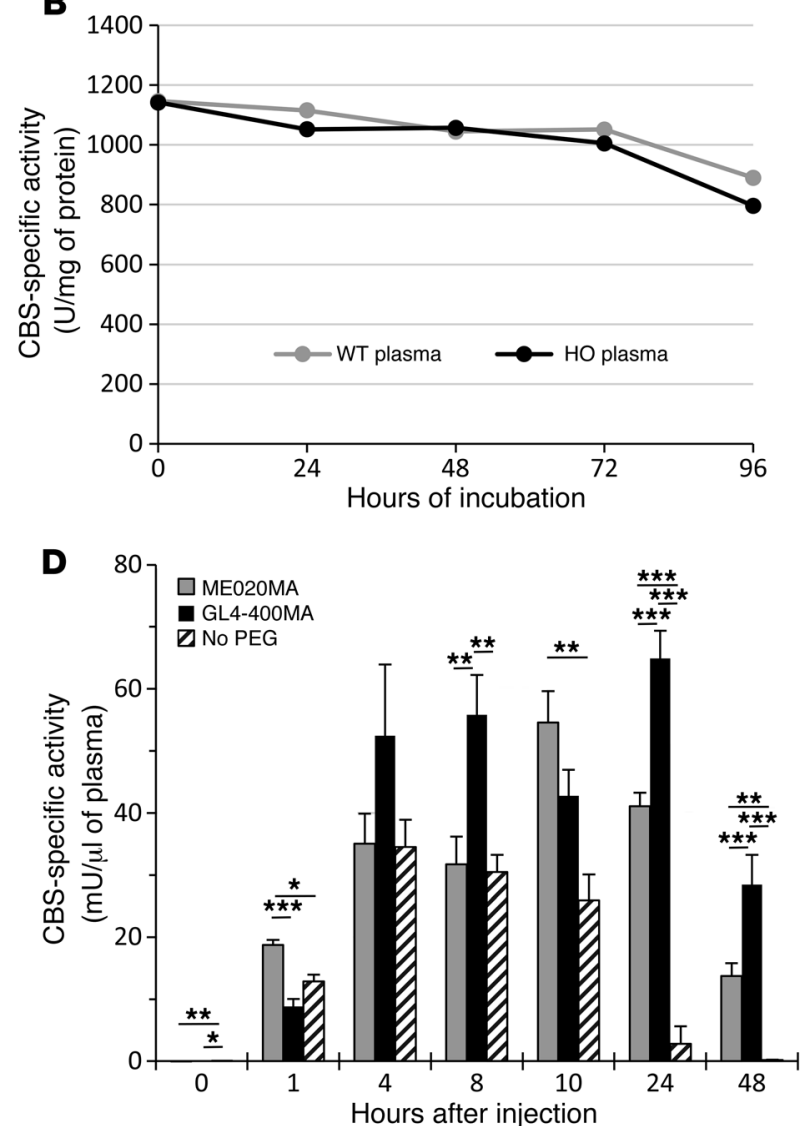

$\mathbf{E}$

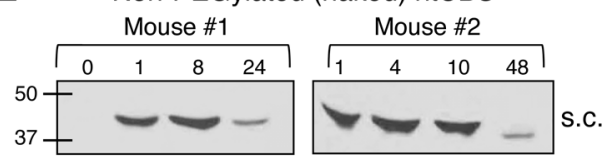

PEGhtCBS (GL4-400MA)

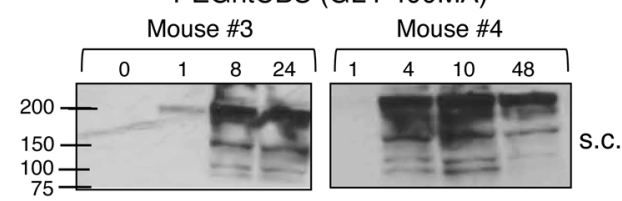

Figure 1. Enzyme retention time in vivo is enhanced following htCBS PEGylation. (A) CBS activity in plasma taken from C57BL/6) mice that were injected with $5 \mathrm{mg} / \mathrm{kg} \mathrm{BW}$ of htCBS via the i.p., i.v., or s.c. route. Two experimental arms ( $n=5$ each) were used for each injection route (due to an IACUC restriction on blood volume collected and time between different bleedings of the same mouse; the 1-hour time point was chosen as a shared point between all groups for comparison). Blood was collected after injection from the mice in group 1 at $0,1,8$, and 24 hours and from the mice in group 2 at $1,4,10$, and 48 hours. (B) The htCBS enzyme was incubated in WT or HO plasma at $37^{\circ} \mathrm{C}(160 \mathrm{ng} / \mu \mathrm{l})$, and activity was measured at the indicated time points. (C) Coomassie-stained SDS-PAGE of the PEGylated and non-PEGylated htCBS with the corresponding specific activity values. (D) Mice $(n=4-5)$ were injected s.c., as described in A, with ME020MA- or GL4-400MA-PEGylated htCBS as compared with the non-PECylated htCBS. (E) Plasma samples from mice injected s.c. with non-PEGylated htCBS or GL4-400MA PEGylated htCBS were analyzed by Western blotting using an anti-hCBS Ab. Data in $\mathbf{A}$ and $\mathbf{D}$ are presented as the mean \pm SEM and were compared using ANOVA, followed by Tukey's post-hoc test. ${ }^{*} P \leq 0.05,{ }^{* *} P \leq 0.01$, and ${ }^{* * *} P \leq 0.001$. PEG, PEGylated; No PEG, non-PEGylated.

PEGylation enhances the plasma half-life of htCBS in vivo. Conjugation of PEG to biopharmaceuticals has emerged as the most promising technique to positively impact the pharmacokinetics of a variety of parenterally administered substances. Accordingly, and since the modification of cysteine residues with maleimide-activated PEG is specific, simple, and forms a biologically inert thioether bond, we monitored the effect of cysteine-directed PEGylation of the enzyme on activity in vitro and on retention time in vivo (see also Supplemental Figure 2A, which shows most of the cysteine-directed PEG types used, presented with good retention times in vivo). For that purpose, htCBS was PEGylated with a low 2-kDa molecular-weight linear PEG (designated ME020MA, to generate the ME020MA PEGhtCBS) or a high 40-kDa molecular-weight, 4-arm branched PEG (designated GL4-400MA, to generate the GL4-400MA PEGhtCBS). PEGylation did not affect the specific activity of the enzyme (Figure 1C). A dose of $5 \mathrm{mg} / \mathrm{kg}$ BW PEGylated htCBS (PEGhtCBS) was administered to C57BL/6 J mice via the s.c. route, and CBS activity was monitored at different time points. The PEGylated enzymes showed significant activity in the circulation 24 and 48 hours after injection. As shown in Figure 1D, ME020MA PEGhtCBS showed specific activity of 41 and $14 \mathrm{mU} / \mu \mathrm{l}$ plasma, and GL4-400MA PEGhtCBS showed specific activity of 65 and $28 \mathrm{mU} / \mu \mathrm{l}$ plasma 24 and 48 hours after injection, respectively. As shown in Figure 1E for GL4-400MA 


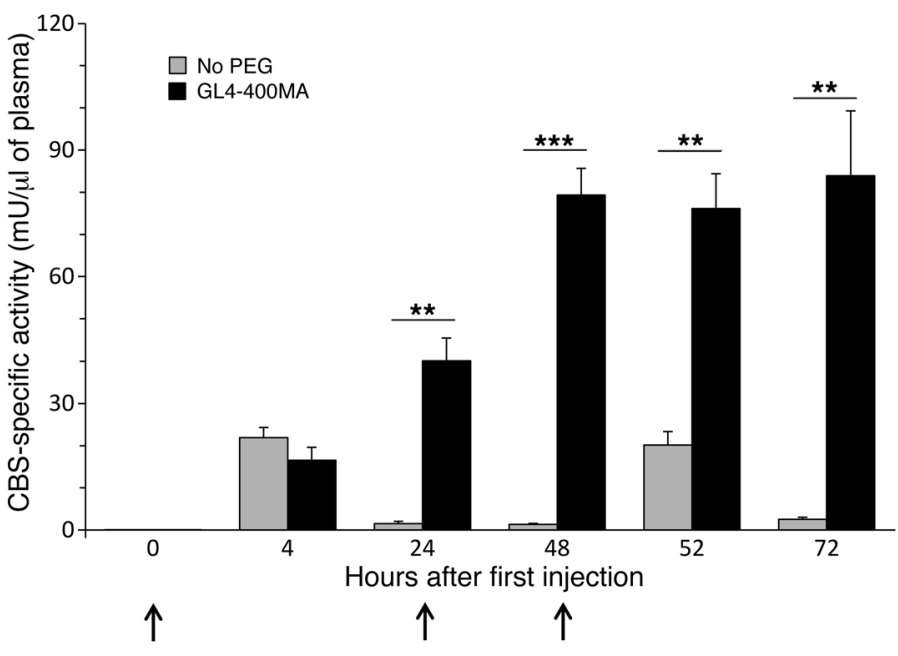

PEGhtCBS, the activity in plasma was significantly extended for the PEGylated protein as compared with that of the non-PEGylated counterpart. Figure 1E, together with Supplemental Table 1 , thus further attests to the rapid clearance of the non-PEGylated enzyme, supporting rapid clearance as the main mechanism for loss of activity of this enzyme.

As shown in Supplemental Table 2, the half-life increased from 2.7 hours for the non-PEGylated htCBS to 16.7 and 30.4 hours for htCBS PEGylated with ME020MA and GL4-400MA, respectively. This means that the time of exposure after a single dose would be 5- to 10-fold longer for the PEGylated forms. Additionally, the bioavailability after s.c. administration ranged from $50 \%$ to $80 \%$, suggesting that the s.c. route is a reasonable one for clinical studies.

A repeated injection regimen was also tested. C57BL/6J mice were injected 3 times with $5 \mathrm{mg} / \mathrm{kg} \mathrm{BW}$ of htCBS on 3 consecutive days, and blood samples were collected every 24 hours, as well as after 4 hours after the first and third injections. Figure 2 shows an activity peak of $20 \mathrm{mU} / \mu$ l plasma 4 hours after injection. However, 24 hours after each injection, only residual activity could be demonstrated for the non-PEGylated enzyme, whereas for the GL4-400MA PEGhtCBS, significantly higher activity levels (up to $84 \mathrm{mU} / \mu \mathrm{l}$ plasma) for a substantial period of time were observed, demonstrating the superiority of the PEGylated enzyme compared with its non-PEGylated counterpart.

PEGhtCBS administration positively impacts plasma levels of total homocysteine and cystathionine concentrations and restores normal cysteine levels. The ultimate goal for the use of htCBS to treat CBSDH is to lower the toxic total homocysteine (tHcy) load and elevate cystathionine and cysteine levels. These changes are expected to prevent, reverse, or delay the onset of CBSDH symptoms. Homocystinuria research, however, has long been hindered by the lack of a suitable animal model. Mice with a completely knocked-out CBS gene die within 2 to 3 weeks after birth. The human-only (HO) mice, which were previously generated in our laboratory, are different in that, in addition to being KOs for the mouse gene, they are transgenic for the human gene and express low levels of hCBS, which allows their survival to adulthood (21). HO mice exhibit severe elevations of tHcy, methionine, S-adenosylmethionine, and S-adenosylhomocysteine and present with
Figure 2. Repeated injection of the PEGylated, but not the non-PEGylated, htCBS shows buildup of CBS activity in vivo. [57BL/6] mice were injected at $0,24,48$ hours (indicated by arrows) with $5 \mathrm{mg} / \mathrm{kg}$ BW of the non-PEGylated htCBS $(n=5)$ or with GL4-400MA PEGylated htCBS $(n=5)$ and were bled at the indicated time points. Data are presented as the mean \pm SEM and were compared using an unpaired Student's $t$ test. ${ }^{* *} P \leq 0.01$ and ${ }^{* *} P \leq 0.001$. a concomitant decrease in plasma and hepatic levels of cysteine. Accordingly, these mice exhibit characteristics that, in several aspects, recapitulate the disease as it occurs in humans.

Since diurnal variation may obscure the htCBS-dependent metabolic changes, we followed the transsulfuration metabolites throughout a 24-hour cycle. Figure 3A shows that tHcy levels changed considerably, and thus it was decided to administer the enzyme and perform blood collections at the time of the lowest tHcy levels (15:00), when the effect of the enzyme could be isolated from the natural tHcy clearance mechanisms (data for each mouse are presented in Supplemental Figure 1 for all metabolites).

In order to evaluate the impact of the administered PEGhtCBS on sulfur amino acid metabolites in vivo, we treated HO mice with either $5 \mathrm{mg} / \mathrm{kg}$ BW of GL4-400MA PEGhtCBS or the non-PEGylated enzyme for 5 consecutive days. Treatment was then stopped for 10 days and restarted on day 14 for an additional 5 consecutive days of once-daily injections. Figure $3 \mathrm{~B}$ describes the changes in tHcy levels for individual $\mathrm{HO}$ mice, and Figure $3 \mathrm{C}$ depicts the average tHcy and cystathionine levels. Initial tHcy levels varied greatly among individual HO mice (100-285 $\mu \mathrm{M})$. However, once treated with the PEGylated enzyme, a significant drop in tHcy plasma concentrations (up to a $250 \mu \mathrm{M}$ reduction, representing an $87 \%$ drop for mouse 435 ) was apparent and was remarkably similar for all mice treated. As shown in Figure 3C, the mean concentration of tHcy 24 hours after the second injection was $55 \mu \mathrm{M}$, a $70 \%$ decrease from the initial $181 \mu \mathrm{M}$ concentration levels. Similarly, tHcy levels were in the range of $38 \mu \mathrm{M}$ to $45 \mu \mathrm{M} 24$ hours after the fourth and fifth injections, respectively. A blood sample collected on day 11 demonstrated that the effect was not completely diminished 7 days after the last injection, with tHcy values at $112 \mu \mathrm{M}(62 \%$ of the initial value). On day 14 , tHcy levels were approaching pretreatment levels, and renewing the injection regimen for an additional 5 consecutive days resulted in a drop of tHcy levels to $60 \mu \mathrm{M}$. Cystathionine levels were also greatly increased following the injections, inversely reflecting the changes in tHcy levels. As shown in Figure 3C, whenever tHcy levels were reduced, cystathionine levels were increased and vice versa. Cystathionine levels rose to as high as $43 \mu \mathrm{M}$ ( 24 hours after the fifth injection; day 5), representing an approximately 9 -fold increase from the 

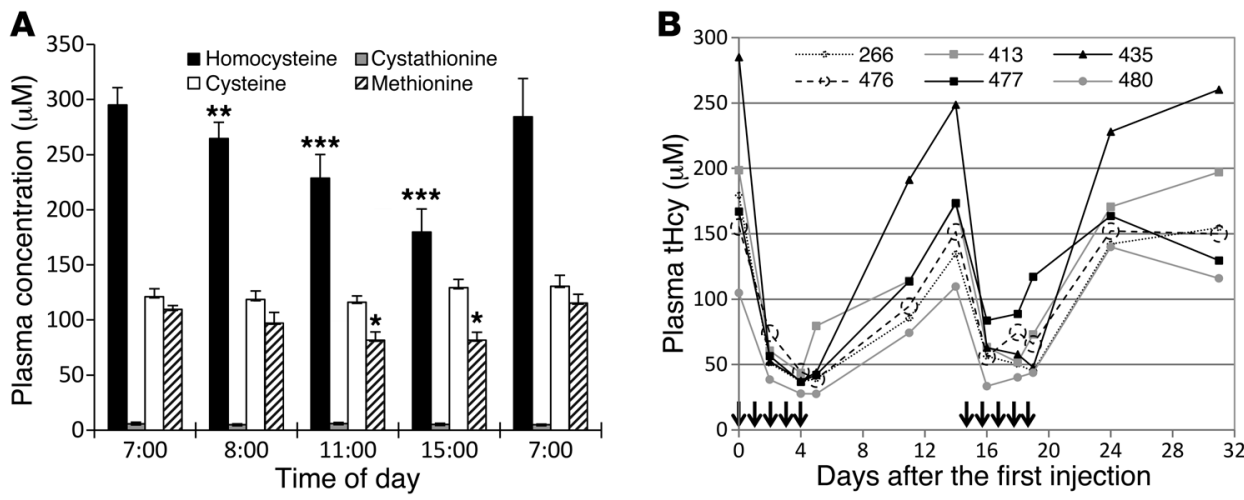

Figure 3. Repeated injection of PEGhtCBS significantly impacts tHcy, cystathionine, and cysteine plasma levels. (A) Metabolites levels in $\mathrm{HO}$ mice $(n=6)$ throughout a 24-hour cycle. (B) tHcy levels in individual $\mathrm{HO}$ mice $(n=6)$ that were injected with $5 \mathrm{mg} /$ kg PEGhtCBS for 5 consecutive days on weeks 1 and 3. (C) Mean tHcy and cystathionine levels of the animals described in B. See Figure 4 for typical levels of metabolites in untreated $\mathrm{HO}$ mice. (D) Mean tHcy and cystathionine levels in HO mice $(n=5)$ that were injected with $5 \mathrm{mg} / \mathrm{kg}$ non-PECylated htCBS for 5 consecutive days on weeks 1 and 3 . (E) Comparison of cysteine levels in the animals injected with PEGylated versus non-PEGylated htCBS. Data in A, C, and D are presented as the mean \pm SEM and are compared with time 0 values, using a paired Student's $t$ test. Data in $\mathbf{E}$ are presented as the mean $\pm \mathrm{SEM}$, and each time point is compared between the 2 groups using an unpaired Student's $t$ test. ${ }^{*} P \leq 0.05,{ }^{* *} P \leq 0.01$, and ${ }^{* *} P \leq 0.001$. Arrows in panels B-E represent injection times.
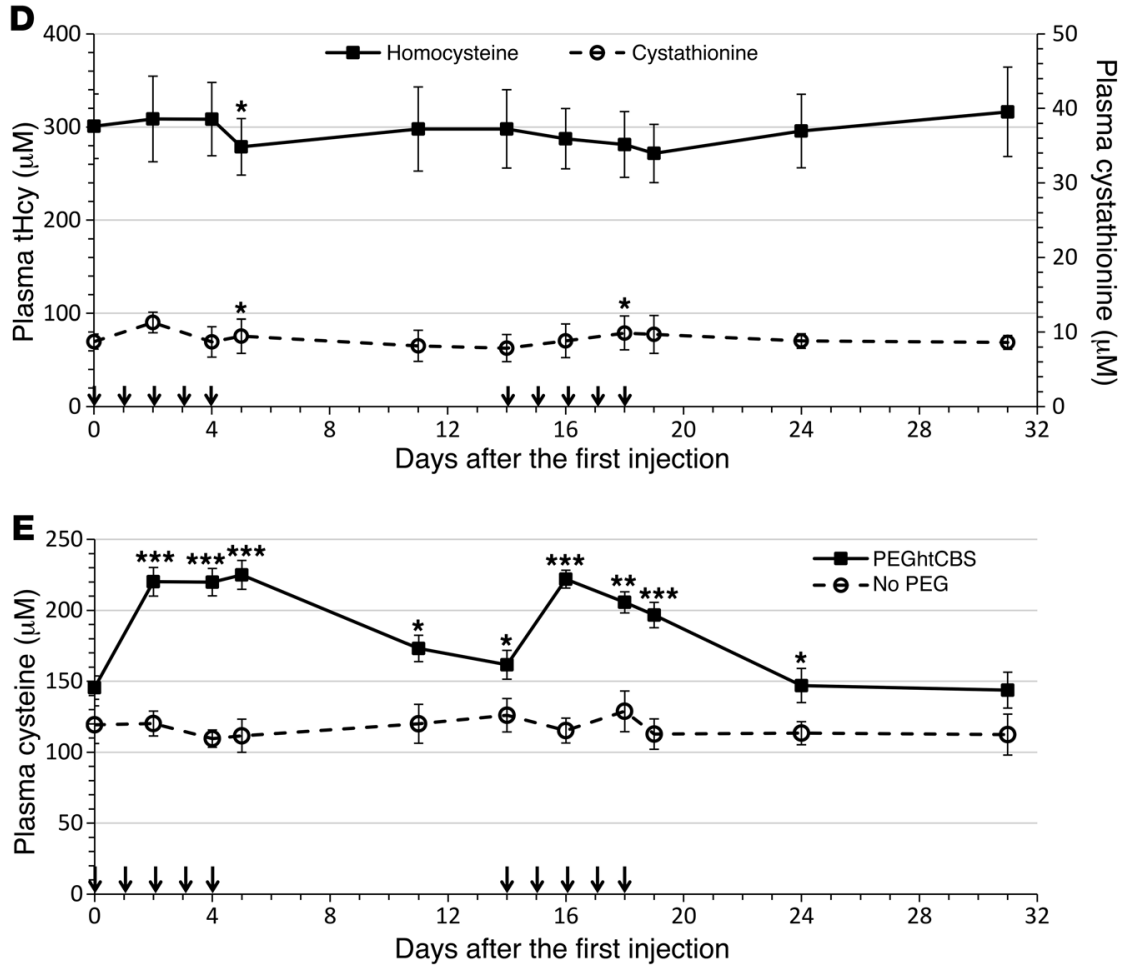

initial $4.8 \mu \mathrm{M}$ at time 0 (the reference ranges for cystathionine in WT and $\mathrm{Cbs}^{+/-}$heterozygous mice are $2.5 \pm 0.3 \mu \mathrm{M}$ and $5.0 \pm$ $0.8 \mu \mathrm{M}$, respectively) (22). Importantly, as shown in Figure 3E, treatment with PEGhtCBS normalized the cysteine levels, starting at $145 \mu \mathrm{M}$ and rising to $225 \mu \mathrm{M}$ during the treatment periods. In contrast to the effect of the PEGylated enzyme, no changes in tHcy or cystathionine levels were observed upon administration of non-PEGylated htCBS (Figure 3, D and E).
Although using the GL4-400MA PEGylated enzyme resulted in a significant improvement in biochemical changes, this specific PEG is more complex in nature (4 arms), expensive, and, to our knowledge, has not been used in any approved drug. Accordingly, we tested several different PEG chemistries (Supplemental Figure 2), and our data indicate that CBS PEGylated with PEGs of lower than 20-kDa molecular weight demonstrated a more rapid loss of CBS activity in plasma compared with CBS enzymes modified 

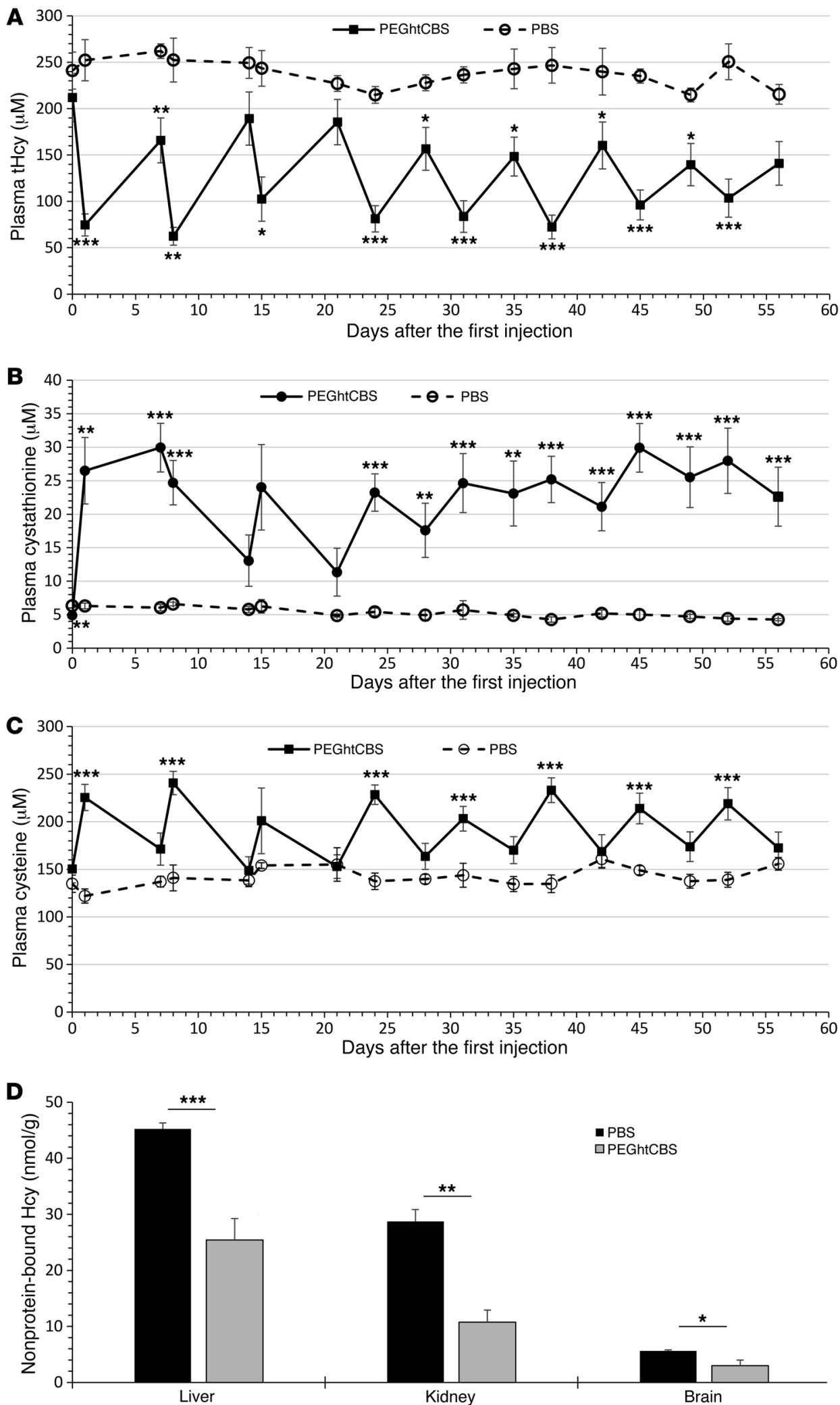

Figure 4. Long-term repeated injections of PEGhtCBS significantly impacts tHcy, cystathionine, and cysteine plasma levels. (A-C) tHcy, cystathionine, and cysteine plasma levels in $\mathrm{HO}$ mice that were injected with $7.5 \mathrm{mg} / \mathrm{kg} \mathrm{BW}$ of PEChtCBS $(n=8)$ or PBS $(n=4)$ twice per week (Monday and Thursday) for the first 2 weeks and 3 times per week (Monday, Wednesday, and Friday) for an additional 6 weeks. Values indicate plasma levels 24 hours (Tuesdays) and 72 hours (Mondays) after injection. (D) Levels of nonprotein-bound homocysteine (Hcy) in liver, kidney, and brain tissues harvested from the long-terminjected animals. Data are presented as the mean $\pm \mathrm{SEM}$, and each time point is compared between the 2 groups using an unpaired Student's $t$ test. ${ }^{*} P \leq 0.05$, ${ }^{*} P \leq 0.01$, and ${ }^{* * *} P \leq 0.001$ with PEGs of higher molecular weight. For that reason, we opted to continue with the linear 20-kDa PEG, designated ME200MAOB (Supplemental Figure 2B). In addition, from a drug manufacturing perspective, this PEG yields a preparation of lower viscosity as compared with that of the $40-\mathrm{kDa}$ PEGs and is cheaper.
Next, we monitored the long-term effect of PEGhtCBS administration. The enzyme was administered to $8 \mathrm{HO}$ mice twice per week for the first 2 weeks and then 3 times per week for the remaining 6 weeks. The dose was increased to $7.5 \mathrm{mg} / \mathrm{kg} \mathrm{BW}$ in order to maximize the effect. Blood samples were collected 24 
A

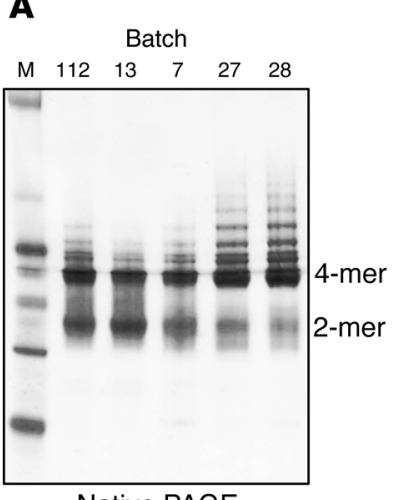

Native PAGE
B htCBS
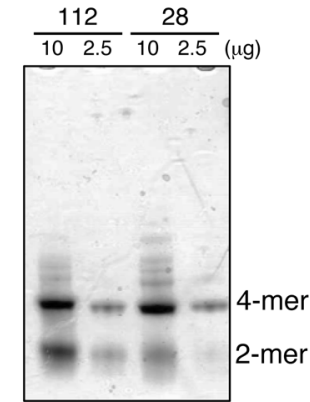

Native PAGE and in-gel activity staining

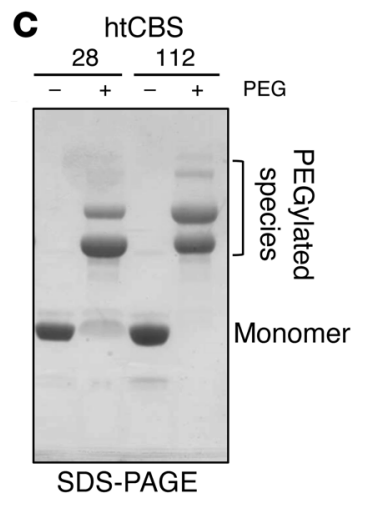

D
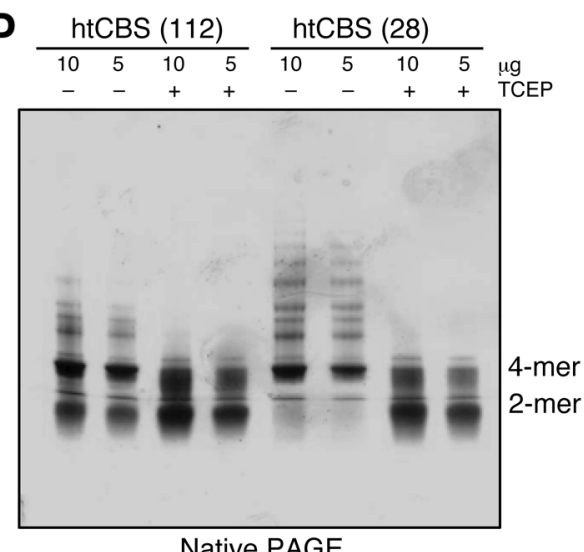

Native PAGE
E

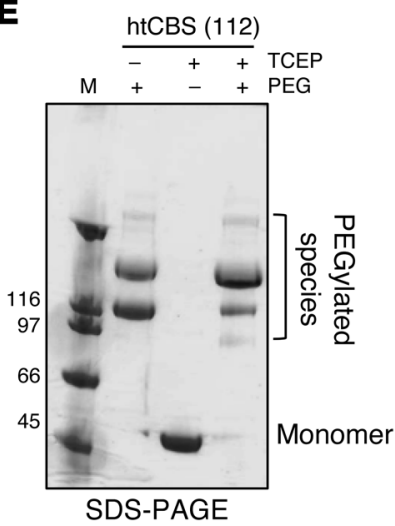

$\mathbf{F}$

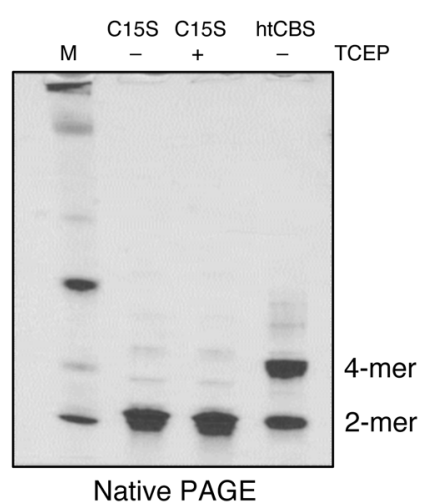

G
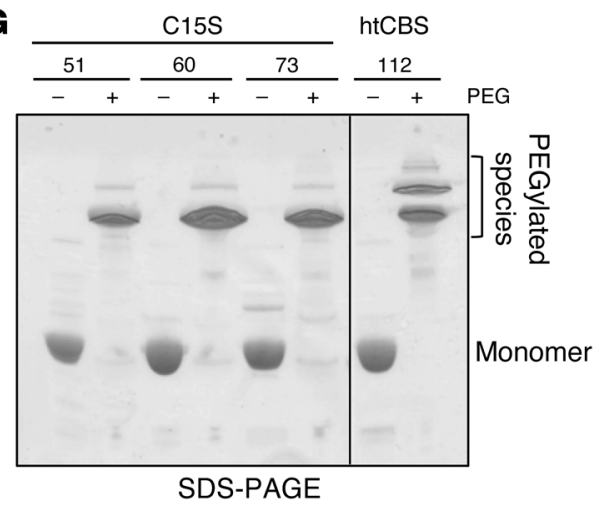

C15S
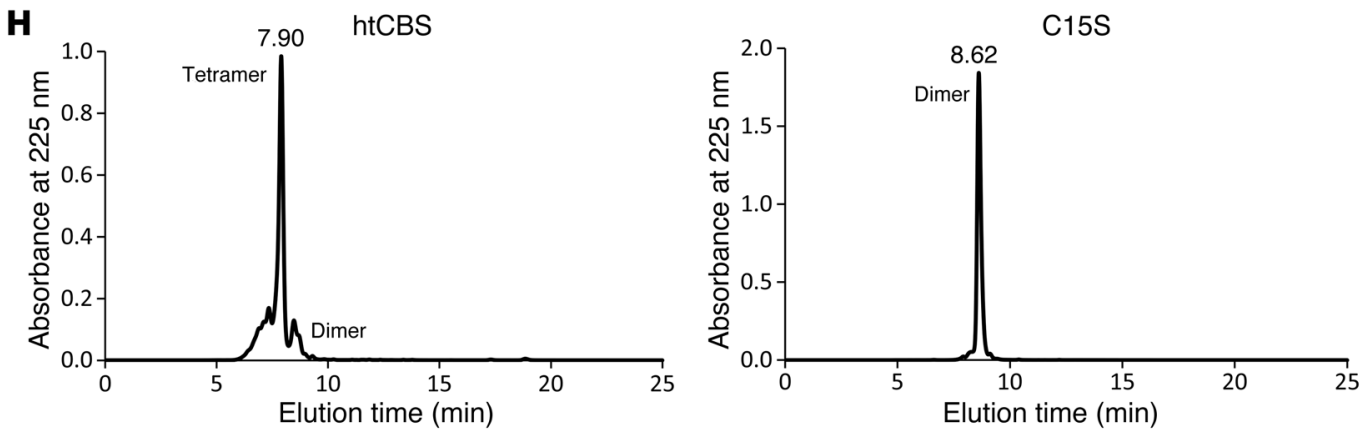

Figure 5. HtCBS C15S mutant prevents protein aggregation, forms mainly dimers, and exhibits a reproducible PEGylation pattern. (A) Coomassiestained native PAGE of different htCBS batches. (B) In-gel activity assay for htCBS. (C) Coomassie-stained SDS-PACE showing a different PEGylation pattern for 2 independent batches of htCBS. (D) Coomassie-stained native PAGE of 2 different htCBS batches, with or without TCEP. (E) Coomassie-stained SDS-PAGE showing the effect of TCEP on PEGylation. (F) Coomassie-stained native-PAGE showing the difference between htCBS C15S and the htCBS dimer/tetramer ratio. (C) Coomassie-stained SDS-PACE showing the reproducibility of htCBS C15S PEGylation between different batches and comparison with htCBS. (H) SEC-HPLC showing a predominantly tetrameric htCBS and an exclusively dimeric htCBS C15S mutant. M, molecular weight marker.

hours after the first weekly injection and 72 hours after the last weekly injection. As shown in Figure 4A, plasma tHcy levels were reduced from $212 \mu \mathrm{M}$ at time 0 to an average in the range of 62 to $103 \mu \mathrm{M} 24$ hours after the injections and were within a range of 141 to $189 \mu \mathrm{M} 72$ hours after the last weekly injection. From day 28 on, the 72-hour post-injection values were significantly lower than the time 0 value $(P \leq 0.02)$. Cystathionine and cysteine levels were positively influenced as well. Cystathionine levels increased from $6 \mu \mathrm{M}$ to as high as $30 \mu \mathrm{M}$ (Figure $4 \mathrm{~B}$ ), and cysteine levels were constantly above $200 \mu \mathrm{M} 24$ hours after injection (Figure 4C). From day 24 on, fluctuations in cystathionine concentrations were much less pronounced compared with those observed in the first 3 weeks of treatment. We also analyzed liver, kidney, and brain tissues to determine the levels of nonprotein-bound homocysteine (free and disulfide-linked forms), known to be significantly elevated in $\mathrm{HO}$ mice compared with the 1-5 nmol/g levels detected in WT mice (22). As shown in Figure 4D, a significant reduction of $44 \%, 63 \%$, and $47 \%$ in nonprotein-bound homocysteine was detected in liver, kidney, and brain tissues, respectively.

A C15S-mutated form of htCBS exhibits no aggregation and a uniform PEGylation pattern. Although it was previously reported that htCBS is less prone to aggregation as compared with the full- 
A

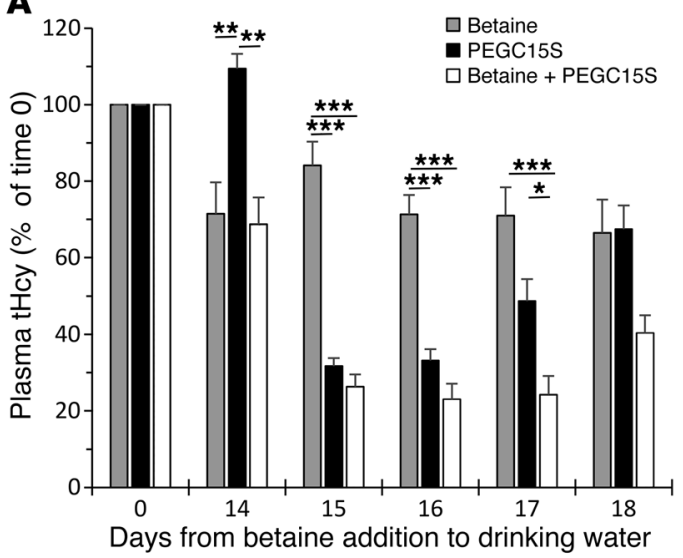

B

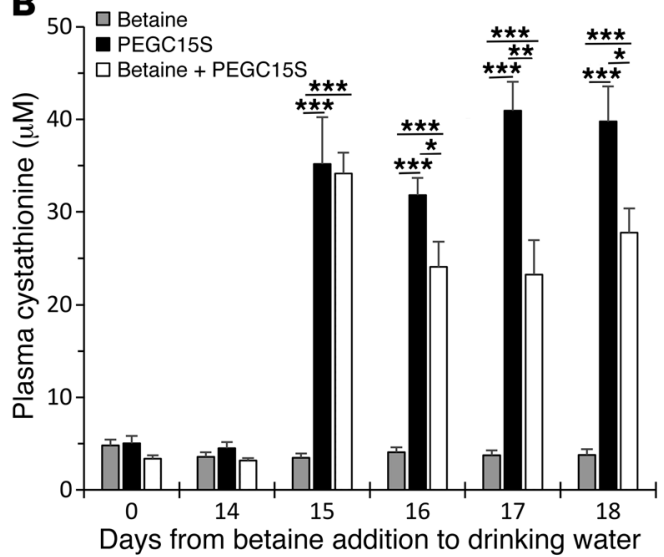

Figure 6. CBS and betaine operate synergistically to reduce and maintain low tHcy levels. (A and B) One group of HO mice was maintained on $2 \%$ betaine water for 18 days (Betaine, $n=5$ ); the second group (Betaine + PEGC15S, $n=5$ ) received the same treatment, but they received 2 injections of $7.5 \mathrm{mg} / \mathrm{kg}$ PEGC15S on days 14 and 15. The third group of mice (PEGC15S, $n=5$ ) was maintained on normal water and received 2 injections of $7.5 \mathrm{mg} / \mathrm{kg}$ htCBS C15S on days 14 and 15. tHcy and cystathionine levels in these groups were determined on day 0 and on days $14-18$. Data are presented as the mean \pm SEM and were analyzed using ANOVA, followed by Tukey's post-hoc test. ${ }^{*} P \leq 0.05,{ }^{* *} P \leq 0.01$, and ${ }^{* * *} P \leq 0.001$.

length protein (17), it still forms tetramers and higher aggregates. Such soluble aggregates may stimulate immune defense mechanisms and lead to, e.g., amyloid deposition (23), but also represent a technological challenge for process development. Batch-to-batch variability, multiple forms as a result of oligomerization or aggregation, and inconsistent PEGylation represent major difficulties for drug development efforts. As shown in Figure 5A, different batches of htCBS showed different dimer/tetramer ratios, with varying degrees of higher aggregates. An in-gel activity assay demonstrated that these aggregates had CBS activity (Figure 5B). Aggregation causes variable PEGylation patterns, as shown in Figure 5C. PEGylation resulted in 2 distinct bands, presumably reflecting a varying degree of PEGylation, as aggregation may obscure the available PEGylation sites. Accordingly, because of a more pronounced aggregation of batch 28 (Figure 5A), htCBS PEGylation resulted in a more pronounced lower band, i.e., fewer PEGylated species, on SDS-PAGE (Figure 5C), whereas PEGylation of batch 112, composed mostly of tetramers and dimers (Figure $5 \mathrm{~A}$ ), resulted in a more pronounced upper band as compared with that observed in batch 28 (Figure 5C). Incubation of both enzyme batches with the reducing agent tris(2-carboxyethyl)phosphine (TCEP) converted many of the tetramers and higher aggregates to a dimeric form (Figure 5D). These data imply that aggregation is driven by the formation of disulfides between exposed cysteines on the surface of htCBS dimers. Thus, we hypothesized that TCEP treatment may also result in a more extensive PEGylation, as more dimers are generated from higher-molecular-weight forms, exposing additional potential PEGylation sites. Indeed, as shown in Figure 5E, the equilibrium between the upper and lower PEGylation bands on an SDS-PAGE was significantly shifted toward the upper band following TCEP treatment as compared with PEGylation in the absence of TCEP. In order to gain more control and reproducibility in the PEGylation process, we mutated the $\mathrm{C} 15$ residue, which was previously shown to be the most reactive cysteine on the surface of CBS (17), to serine, thereby generating the C15S htCBS mutant. Figure 5F depicts a Coomassie-stained native gel, in which 2 main bands (dimers and tetramers) were observed for htCBS, whereas the C15S htCBS mutant had a single band, which, as expected, was not affected by treatment with TCEP. This observation was further substantiated by size-exclusion HPLC (SEC-HPLC). The single peak for the dimeric C15S htCBS mutant eluted at $8.62 \mathrm{~min}$ utes, while the main peak for the htCBS eluted at 7.9 minutes, corresponding to a tetramer (Figure 5H). As shown in Figure G, the absence of aggregates in the C15S htCBS resulted in a more consistent PEGylation pattern between different batches with, almost exclusively, a single PEGylated species appearing on SDS-PAGE. Direct comparison between PEGhtCBS and the PEGylated htCBS C15S (PEGC15S), both in vivo and in vitro (see Supplemental Figure 3 and also compare Figure 6, A and B, and Figure 4, A-C, with Figure 3, $\mathrm{C}$ and $\mathrm{E}$ ), showed no difference in efficacy.

We also tested the effect of PEGC15S at 1, 4, and 24 hours after injection (Supplemental Figure 4). Interestingly, whereas the effect on tHcy and cysteine was observed only 24 hours after injection (Supplemental Figure 4, A and C), a significant increase in cystathionine was already observed at 1 and 4 hours after injection (Supplemental Figure 4B).

PEGC15S and betaine operate synergistically in vivo. It is reasonable to assume that ERT for homocystinuria, once approved, will be administered on a background of protein restriction and betaine supplementation. Thus, it is important to determine the effect of ERT therapy combined with betaine. Accordingly, we compared the effect of betaine, PEGC15S, and a combination of betaine plus PEGC15S treatments on metabolites of the transsulfuration pathway.

The betaine-only and betaine-plus-PEGC15S groups were treated with betaine throughout the 18 days of the experiment, and the latter group was injected with PEGC15S on days 14 and 15. Mice in the PEGC15S-only treatment group were maintained on water with no betaine and injected on days 14 and 15 as well. As shown in Figure 6A, 14 days of betaine treatment resulted in a $29 \%$ and $32 \%$ decrease in tHcy for the betaine-treated groups. The betaine-only treatment group maintained these levels of tHcy out to day 18. Injection of PEGC15S on the background of 
A

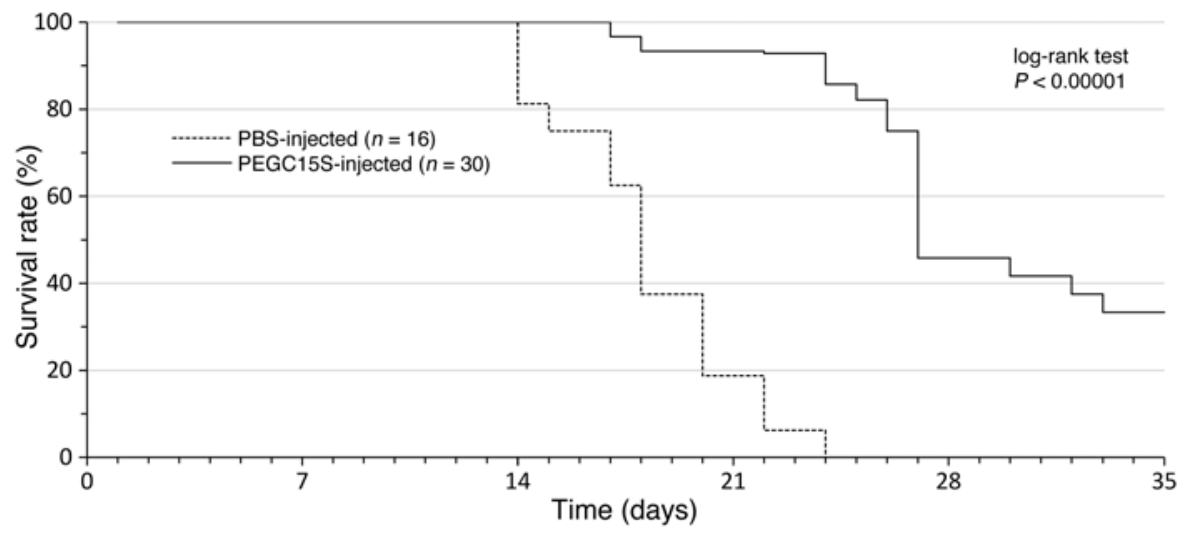

B

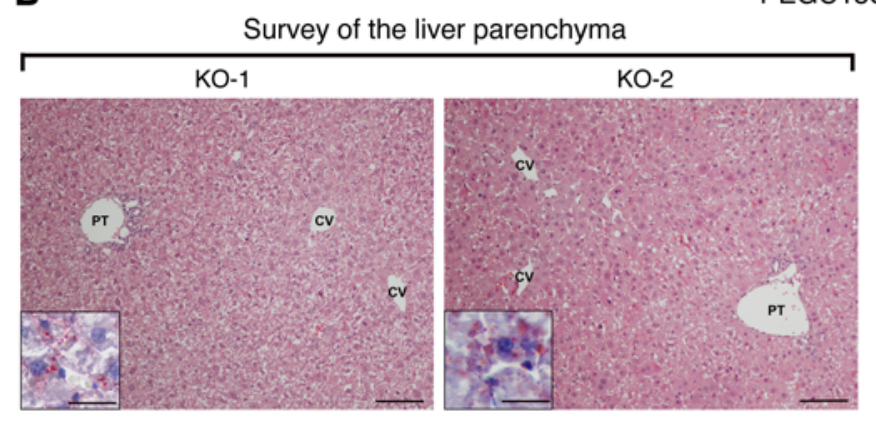

PEGC15S-injected

Survey of the liver parenchyma
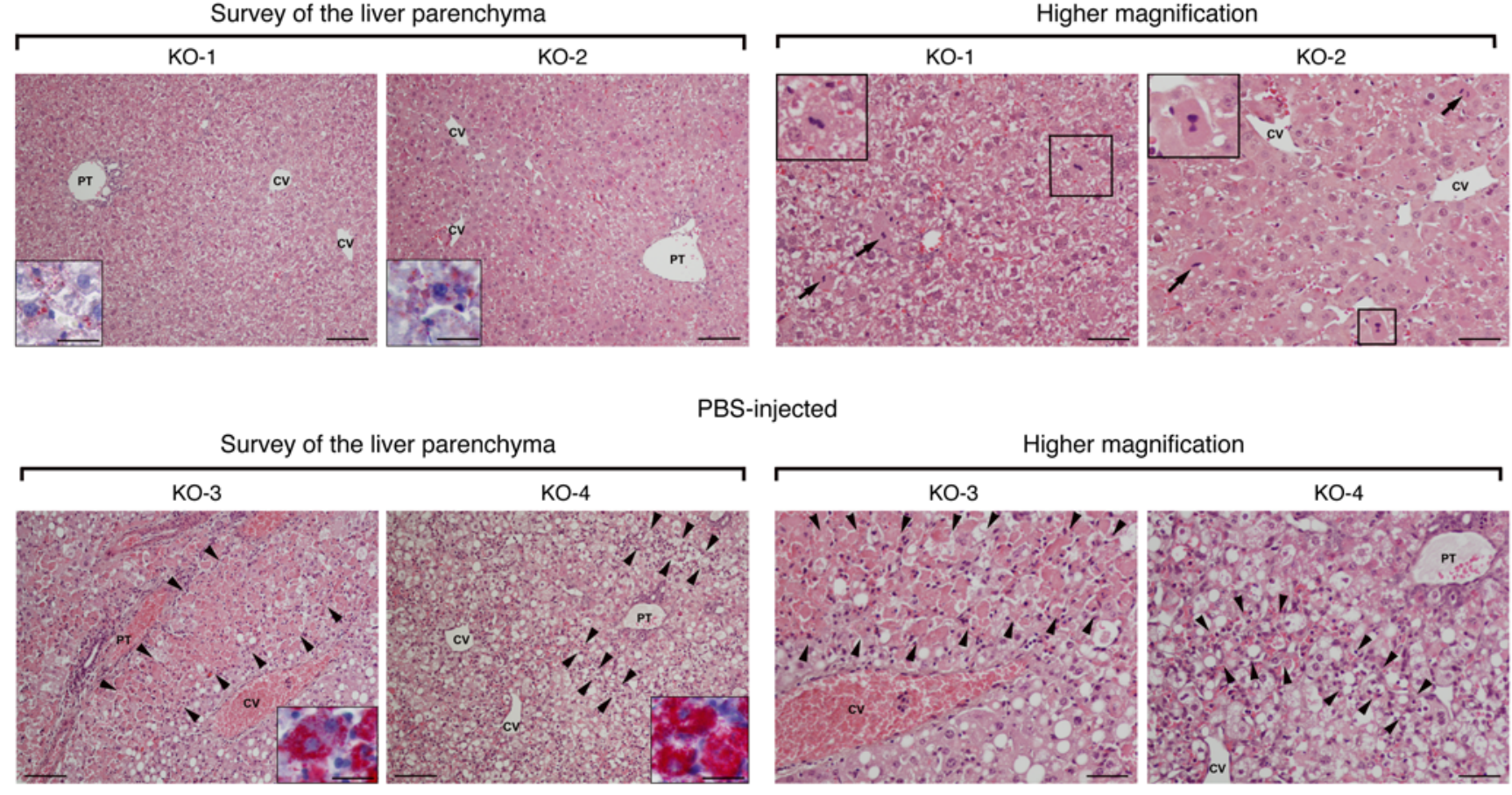

Figure 7. PECC15S administration rescues complete CBS-KO mice from early lethality. (A) Kaplan-Meier survival curve of KO mice that were injected twice weekly with PECC15S $(n=30)$ versus mice injected with PBS $(n=16)$. Mice were maintained on betaine water until day 21. A log-rank test was used for comparison of the 2 survival curves, and significance was calculated using the $\chi^{2}$ test. (B) Liver histology results for 2 PEGC15S-injected KO mice that were sacrificed on day 35 versus liver histology results for 2 PBS-injected KO animals that died on days 17 and 24 (H\&E staining). Low-magnification views of the liver parenchyma (left panels) show moderate changes, with slightly irregular liver cell plates and mild-to-moderate steatosis, in PEGC15S-injected KO-1 and KO-2 mice, contrasting with massive zonal necroses of hepatocytes (KO-3 mouse, marked by arrowheads) and diffuse steatosis, with multiple dispersed hepatocellular necroses (KO-4 mouse, necroses marked by arrowheads) in the PBS-injected KO mice. A specific stain for apolar lipids in the cytoplasm of hepatocytes is shown in the insets. Higher-magnification views (right panels) demonstrate the presence of frequent mitoses (marked by arrows and shown in detail in the insets) and enlarged pleiomorphic nuclei, with prominent nucleoli in hepatocytes, in the PEGC15S-injected KO mice. Higher-magnification views of the liver parenchyma in PBS-injected KO mice demonstrate confluent hepatocellular necroses, with a sparse inflammatory infiltration (KO-3 mouse, marked by arrowheads) or multiple dispersed necroses, accompanied by a prominent resorptive inflammatory reaction (KO-4 mouse, marked by arrowheads). The remaining liver parenchyma show micro- and macrovesicular steatosis in PBS-injected KO mice. CV, central vein; PT, portal tract. Scale bars: $200 \mu \mathrm{m}$ (low-magnification images); $100 \mu \mathrm{m}$ (high-magnification images); and $50 \mu \mathrm{m}$ (insets).

betaine resulted in a more pronounced reduction of $74 \%, 77 \%$, $76 \%$, and $60 \%$ on days $15,16,17$, and 18 , respectively. Treatment with PEGC15S alone resulted in a reduction of $69 \%, 67 \%, 52 \%$, and $33 \%$ on days $15,16,17$, and 18 , respectively. According to these data, injection of PEGC15S with or without betaine supplementation was far superior to betaine treatment alone. In addition, the combination of betaine plus PEGC15S was superior to treat- ment with PEGC15S alone. As shown in Figure 6A, the difference between these 2 groups became statistically significant 48 hours after the second injection (on day $17 ; P \leq 0.05$ ). Thus, we conclude that the combination of PEGC15S and betaine allows the maintenance of lower tHcy levels for an extended period of time.

Betaine treatment did not result in an increase in cystathionine levels over the 18-day experimental period (Figure 6B). In contrast, 
an increase in these levels was observed for the 2 groups that were treated with PEGC15S (without or with betaine). Interestingly, the combination of PEGC15S plus betaine resulted in an increase in cystathionine levels, which, starting on day 16 and beyond, were significantly lower than those in the group that received only the PEGC15S enzyme. This is likely due to the fact that treatment with PEGC15S alone channels homocysteine to condense with serine to form cystathionine. Combination with betaine diverts some of the available homocysteine through the remethylation pathway to produce methionine, and thus less homocysteine is available for PEGC15S to be converted to cystathionine.

PEGC15S rescues KO mice from early death and improves liver disease. The majority of CBS-KO mice die within 2 to 3 weeks after birth. Thus, we hypothesized that administration of PEGC15S to KO pups early after birth would rescue them. The mice were injected twice per week with PEGC15S or PBS for 5 weeks. During the first 21 days, the lactating mothers had drinking water containing $2 \%$ betaine (an estimated daily intake of $4 \mathrm{~g} / \mathrm{kg}$ ). As shown in Figure 7A, on day 21 , only $18 \%$ of the mice survived in the PBSinjected group as opposed to $93 \%$ of those that were injected with the enzyme. No PBS-injected animal survived to day 24, while an $86 \%$ survival rate was recorded for the PEGC15S-injected group. From day 24 and beyond, the number of enzyme-injected animals began to decline as well, with $45 \%$ survival on day 29 and $33 \%$ on day 35 (Figure 7A).

It was previously demonstrated that $\mathrm{KO}$ mice suffer from severe liver damage (22). Prolonged survival of KO mice may thus be correlated with improvement in liver function after CBS treatment. Accordingly, we performed histological analysis of liver sections from animals that were injected twice weekly with PEGC15S for a period of 35 days. Animals were sacrificed on day 36 and liver samples processed for histological analysis by optical microscopy. PBS-injected animals did not survive to day 35 , but for 2 animals, we were able to take liver samples for processing immediately after death on days 17 and 24, respectively (Figure 7B). The analyses were carried out in a blinded fashion. PBSinjected animals developed severe hepatopathy characterized by pronounced microvesicular to macrovesicular steatosis and substantial death of hepatocytes, with minimal or no fibrosis. Multiple mono or oligo cellular necroses of hepatocytes dispersed throughout the liver lobule, accompanied by a prominent inflammatory resorptive reaction, were found in 1 animal, whereas massive confluent necroses of hepatocytes mainly in periportal zones with a sparse inflammatory infiltrate dominated a feature in the other. Signs of liver parenchyma regeneration were minimal in both animals. Two PEGC15S-injected animals showed moderate changes in the liver, with minor signs of parenchyma damage, but prominent regeneration, and were correctly recognized by the examiner as the ones receiving therapy. Changes indicative of liver parenchyma regeneration (slightly irregular architecture of the liver lobule, basophilic cytoplasm of hepatocytes, frequent mitoses and binucleated hepatocytes, and nuclear pleiomorphy and enlargement with prominent nucleoli) were the most conspicuous feature. The overall morphology was consistent with that of increased proliferation and transcription in hepatocytes. Furthermore, rare focal necroses of hepatocytes and mild steatosis of a microvesicular type were detected, and fibrosis was minimal.

\section{Discussion}

In systems biology, the robustness of a biological system is defined by its ability to function properly in the face of perturbations. Redundancy of elements in the system is one of the mechanisms by which such robustness is achieved (24). The biological transsulfuration system seems to lack proper redundancy with its components and is therefore prone to mutational perturbations, as one of its elements, the CBS enzyme, is the only component in this system that can process homocysteine to cystathionine. A limited system redundancy partly exists, as homocysteine, the first metabolite that funnels into the pathway, can alternatively be converted to methionine through the remethylation pathway, thus relieving the homocysteine load. In addition, cysteine, a downstream product, can be obtained directly from the diet. Nevertheless, these pathways are limited in their capacity to maintain normal levels of metabolites, and lack of CBS activity manifests with detrimental consequences for patients if left untreated. Thus, a therapeutic approach for $\mathrm{CBSDH}$ must correct the perturbation rather than reenforce the alternative pathways. For most of the $\mathrm{B}_{6}$-nonresponsive $\mathrm{CBSDH}$ patients, the only current option is to reduce the intake of methionine by a strict low-methionine diet supplemented with cysteine in the amino acid mixture and by decreasing the homocysteine concentration with betaine supplementation. Currently, since gene therapy, the ultimate potential cure for metabolic diseases such as CBSDH, is still plagued with safety issues in the form of vector-induced genomic perturbations (insertional mutagenesis, genotoxicity) (25), ERT using htCBS seems to be the best hope for these patients to ease the symptoms of the disease while ameliorating the demanding dietary measures.

ERT requires chronic administration of the pharmacologically active substance, and thus lowered costs and suitability for self-administration are of importance. Therefore, s.c. administration is favored. However, as we show here (Figures 1 and 2), delivering htCBS via the s.c. route not only resulted in lower activity in the circulation compared with that seen with other routes, but, like what was observed with the other routes, showed almost no activity 24 or 48 hours after injection. Therefore, protein modification using PEG, a strategy that has been in the forefront of protein modification in the pharmaceutical industry for the past 25 years (20), was vital to achieve an acceptable retention time. Indeed, PEGylation of the htCBS enzyme improved the pharmacokinetics and, subsequently, the pharmacodynamic profile of the enzyme in vivo (Figures 1-3).

In healthy individuals, tHcy levels are in the range of approximately 5 to $15 \mu \mathrm{M}$ (15), $98 \%$ of which is in the form of disulfides or is protein bound. Only $2 \%$ of the tHcy exists as a nonbound, free, reduced aminothiol that can serve as a substrate for $\operatorname{CBS}(26,27)$. This balance is dramatically altered in CBSDH patients, with free, reduced homocysteine reaching $10 \%-25 \%$ of the tHcy values that are observed in these patients (up to $\sim 400 \mu \mathrm{M}$ ) (28). In both cases, free, reduced homocysteine comprises only a fraction of tHcy. Nevertheless, the data presented in this study show that the presence of PEGylated htCBS in the circulation resulted in a 70-250 $\mu \mathrm{M}(70 \%-90 \%)$ decrease in tHcy levels (Figure 3). Thus, the initial levels of free homocysteine available for the enzyme $(10 \%-$ $25 \%$ of the total) cannot solely account for the decrease in tHcy levels. Therefore, additional pools of free homocysteine likely 
become available to the enzyme, as the balance between bound and unbound homocysteine is affected upon PEGC15S administration. Alternatively, as free homocysteine becomes scarce as a result of the enzyme activity, the balance between free homocysteine and homocysteine adducts (in the form of protein-bound homocysteine or disulfides) changes to favor the generation of free homocysteine. Together, this implies that, upon administration of the PEGylated enzyme, the balance between bound and unbound homocysteine is shifted toward the free homocysteine that is processed by the enzyme in circulation. Thus, the PEGylated enzyme serves as a "sink" that constantly and systematically relieves the flux of homocysteine from extra- and intracellular pools.

It is noteworthy that even a reduction of $70 \%$ to $90 \%$ from the initial tHcy values was not sufficient to restore normal tHcy levels. This raises the question whether the significant drop in tHcy levels is enough to be of therapeutic utility in CBSDH patients. It was previously shown (29) that in mice with at least 54 times higher tHcy levels than those in WT mice, several phenotypic features were observed including facial alopecia, osteoporosis, reduction in mean survival, and other signs. However, no such signs were observed in mice that had tHcy levels of approximately 30 times the normal values. The authors concluded that homocysteine elevation is pathogenic only above a certain threshold level. Of particular interest are the results obtained in a multicenter observational study in humans (30), in which it was found that various treatment combinations failed to restore normal homocysteine levels in $\mathrm{CBSDH}$ patients and that $\mathrm{B}_{6}$ nonresponders had tHcy levels that were 6-8 times higher than the upper limit in the normal population. Nevertheless, these treatment strategies, achieving an imperfect biochemical control, significantly (relative risk, 0.09; $P<0.0001)$ reduced the risk of thromboembolism in these patients. Collectively, these data indicate that in order to ameliorate the clinical manifestation of $\mathrm{CBSDH}$, a reduction of tHcy to below a threshold level, estimated at 80 to $100 \mu \mathrm{M}$, is sufficient.

A low-methionine diet can be effective in establishing and maintaining metabolic control in patients. This effect, however, is hindered by the lack of dietary compliance, particularly among late-diagnosed patients, resulting in a concomitant increase in homocysteine, accompanied by development of symptoms, especially in children (10). Betaine may help to achieve metabolic control in less compliant patients, and its combination with the diet may represent the best available treatment at present. However, current therapies for vitamin $\mathrm{B}_{6}$ nonresponders do not increase cystathionine levels, and cysteine supplementation is warranted. In contrast, the murine data presented in this study show that administration of PEGhtCBS resulted in a significant reduction of tHcy levels, while the mice were maintained on a standard diet of $19 \%$ protein content (as opposed to the low-methionine diet used to treat human patients). This effect was far superior to the effect of betaine alone under these conditions, and combination of the enzyme with betaine showed that both can work synergistically to maintain low tHcy levels for an extended period of time (Figure 6). In addition to achieving metabolic control of homocysteine levels, administration of PEGhtCBS resulted in a significant increase in cystathionine levels and normalization of cysteine levels.

The role of cystathionine, other than serving as a substrate for CGL to produce cysteine, is unknown. Interestingly, CBS-
KO mice suffer severe growth retardation and hepatopathy, and most die within 3 weeks after birth, even while the mothers are on betaine (21). The HO mice, which carry 2 copies of the human CBS gene, have high levels of tHcy but nevertheless suffer only minor consequences, if any. The only difference in metabolites between these 2 murine models is the level of cystathionine, which is significantly elevated in $\mathrm{HO}$ mice $(\sim 10 \mu \mathrm{M})$ as compared with that in KO mice $(\sim 1 \mu \mathrm{M})$, which may imply a positive role for cystathionine. Indeed, it has been suggested (31) that cystathionine may protect against ER stress-induced lipid accumulation, tissue injury, and apoptotic cell death. Moreover, high levels of cystathionine in brain tissues, along with rigorous control over CGL and CBS expression during mammalian development (32), are in line with an important role for cystathionine other than that of a precursor to cysteine. One may thus argue that the clinical manifestations of CBSDH may not only be attributed to high levels of the toxic homocysteine but, additionally, to the lack of cystathionine and cysteine and to elevated methionine levels (33). Low intracellular cystathionine levels may be corrected by uptake of this amino acid via a transporter mechanism such as the cysteine-glutamate transporter (34). Accordingly, the marked increase in cystathionine documented here following administration of PEGhtCBS may also be beneficial in ameliorating CBSDH symptoms.

Importantly, we observed normalization of cysteine levels following administration of the enzyme, which may present another advantage for CBS ERT. Cysteine biosynthesis from cystathionine is catalyzed solely by CGL. The latter is only present inside the cells and does not exist as a plasma protein (data not shown). Additionally, the extracellular cystathionine produced by the injected enzyme may also enter the cells (34) and serve as an intracellular substrate for CGL. In addition, it was recently suggested that a reduction of tHcy levels may impede the formation of cysteinehomocysteine adducts, which are rapidly cleared via urine excretion (35), or the formation of albumin-bound cysteines, thus resulting in higher levels of cysteine in plasma (35). Normalizing cysteine levels without cysteine supplementation may present another advantage of CBS ERT.

The positive effect of CBS administration was not limited to a change in metabolite levels in plasma and tissues, but showed a significant impact on liver disease and survival of the affected mice. It was previously shown that the majority of $\mathrm{KO}$ pups die within 2 weeks after birth and that growth retardation and severe liver disease are the most prominent outcomes of the null CBS condition $(22,36)$. This is in concert with the liver damage documented in this study for the PBS-injected $\mathrm{KO}$ animals and in sharp contrast to the CBS-treated mice, in which signs of liver regeneration substantially dominated over signs of liver parenchyma damage, indicating a capacity of the liver to cope with the metabolic defect.

The data presented in this study provide a proof of concept for PEGhtCBS as a promising ERT for homocystinuria, one that ameliorates the manifestations and metabolic abnormalities that characterize the disease. Clearly, translating a successful academic project into a therapy for human patients requires, among other elements, process upscaling, high-yield production, consistent batches, and pharmacokinetic and toxicological analyses in higher species. These studies were performed successfully and will be published elsewhere. In addition, the successful produc- 
tion, approval, and use in humans of several recombinant enzyme products such as ADAGEN (ERT to treat adenosine deaminase deficiency and SCID), Pulmozyme (to treat cystic fibrosis), pegloticase (to treat gout), and more $(37,38)$, attests to the applicability of enzyme therapies in humans. Our proposed ERT thus provides a therapeutic strategy for CBSDH patients that allows for the prevention or treatment of symptoms and relaxes the need to maintain a strict diet.

\section{Methods}

Construction of an htCBS expression vector. A full-length (551 amino acids) hCBS coding sequence was optimized for bacterial expression and cloned into the pUC57 vector following digestion with the EcoRV restriction enzyme (GenScript USA). The htCBS sequence was then amplified by PCR using primers A1 and A2 (see Supplemental Experimental Procedures for the list of primers used) to generate a sequence coding for the truncated enzyme (amino acids 1-413). The PCR product was then digested with the restriction enzymes NcoI and XhoI and ligated into the pET-28a $(+)$ vector that was digested with the same enzymes. Cloning into the NcoI site of pET-28a(+) results in a $\mathrm{G} \rightarrow \mathrm{C}$ mutation as compared with the native CBS sequence. A Site-Directed Mutagenesis Kit (Agilent Technologies) using primers B1 and B2 was used to restore the WT sequence. The same strategy was used to generate the $\mathrm{C} 15 \mathrm{~S}$ mutant by using primers $\mathrm{C} 1$ and $\mathrm{C} 2$. All sequences were verified by DNA sequencing.

Expression and purification. A detailed description can be found in the Supplemental Experimental Procedures. In short, E. coli BL-21 GOLD (DE3) (Agilent Technologies) or HMS174 (DE3) (EMD Millipore) was transformed with the CBS-encoding plasmid and grown in Terrific Broth. Protein expression was induced at $\mathrm{OD}_{600}=0.6-0.7$ with isopropyl $\beta$-D-thiogalactopyranoside (IPTG) and harvested and lysed 16 hours later. The lysate was then processed through a multistep chromatographic procedure.

PEGylation. Activated PEG molecules were purchased from NOF America, and PEGylation was carried out according to the manufacturer's instructions. For example, coupling of maleimide PEGs to the SH groups of htCBS $(5 \mathrm{mg} / \mathrm{ml})$ was performed in a $100 \mathrm{mM}$ phosphate buffer (pH 6.5) overnight at $4^{\circ} \mathrm{C}$. The molar ratio between PEG and the CBS subunit was either 10:1 or 5:1.

Animals. C576BL/6J and CBS-KO mice were obtained from The Jackson Laboratory. HO mice were previously generated in our laboratory (21). Animals were maintained on the $2920 \mathrm{X}$ extruded standard diet (Envigo). Mice were routinely genotyped by using quantitative realtime PCR as outlined in the Supplemental Experimental Procedures. A single-use lancet for submandibular bleeding was used for blood collection into Capiject T-MLHG lithium heparin (12.5 IU) tubes with gel (Terumo). Tubes were then centrifuged at 1,200 $\times g$ for 10 minutes, followed by collection of plasma into $1.5-\mathrm{ml}$ tubes and storage at $-80^{\circ} \mathrm{C}$.

In-gel CBS activity staining and CBS activity assay. Protein samples were separated using Native Page (Bio-Rad). The gel was then incubated for 15 to 30 minutes in a staining solution (100 mM Tris$\mathrm{HCl}, \mathrm{pH}$ 8.0, 20 mM L-cysteine, $50 \mathrm{mM}$ 2-ME, $0.1 \mathrm{mM}$ PLP, and 0.2 $\mathrm{mM}$ lead nitrate). The reaction was stopped by submerging the gel in $7 \%$ acetic acid.

A detailed description of the CBS activity assay can be found in the Supplemental Experimental Procedures and in ref. 39. In short, CBS activity was determined by a radioisotope assay with ${ }^{14} \mathrm{C}$-labeled serine as a substrate. The ${ }^{14} \mathrm{C}$-cystathionine formed in the reaction was separated from the labeled substrate by overnight descending paper chromatography.

Determination of metabolite concentrations. Plasma metabolites were determined by stable-isotope dilution gas chromatography/mass spectrometry as previously described (40). Measurements of tHcy and nonprotein-bound homocysteine and other aminothiols as well as of amino acids in tissue were performed as described previously $(21,41)$. The analysis was performed in a blinded fashion, without knowledge of the animal treatment regimen.

SEC-HPLC. CBS preparations were separated on a Yarra SEC$3000,300 \times 7.8 \mathrm{~mm}$ SEC column (Phenomenex). The column was calibrated and operated in $100 \mathrm{mM}$ sodium phosphate ( $\mathrm{pH}$ 6.8), at a flow rate of $1 \mathrm{ml} / \mathrm{min}$ at room temperature.

Histology. Liver tissue sections were stained with H\&E to evaluate the histopathological changes. Masson's trichrome staining was performed for detection of fibrosis, and steatosis was verified using Oil Red O staining for detection of apolar lipids in fixed, frozen sections. A detailed description can be found in the Supplemental Experimental Procedures.

Statistics. All data are presented as the mean \pm SEM and were compared using a paired or unpaired, 2-tailed Student's $t$ test. When comparing 3 different experimental arms, data were analyzed by ANOVA, followed by Tukey's post-hoc analysis. A $P$ value of less than 0.05 was considered significant. The log-rank test was used for comparison of survival curves, and significance was calculated using the $\chi^{2}$ test.

Study approval. All animal procedures were approved by the IACUC of the University of Colorado Denver, which is an Association for Assessment and Accreditation of Laboratory Animal Careaccredited (AAALAC-accredited; no. 00235); Public Health Serviceassured (no. A 3269-01); and US Department of Agriculture-licensed (USDA-licensed; no. 84-R-0059) institution.

\section{Author contributions}

EMB designed the studies, conducted the experiments, acquired and analyzed the data, and wrote the manuscript. TM generated preliminary data, provided reagents, participated in the design of the studies, acquired data, performed data analysis, and revised the manuscript. IP managed animal husbandry, assisted with the execution of animal studies, and prepared samples for analyses. RSC developed the purification procedure and provided reagents. $\mathrm{HH}$ performed the histological analysis of tissues and revised the manuscript. JK determined the levels of metabolites in tissues and revised the manuscript. VK coordinated the histological and metabolic analysis of tissues and revised the manuscript. JPK supervised the work, participated in the study design and analysis, and wrote the manuscript.

\section{Acknowledgments}

The authors would like to acknowledge the thousands of sulfur amino acid determinations carried out at this institution in the laboratory of Robert Allen and Sally Stabler. H. Hůlková, J. Krijt, and V. Kožich received institutional support from the Charles University in Prague (PRVOUK-P24/LF1/3 and UNCE 204011) and the Ministry of Health of the Czech Republic (RVO-VFN 64165/2012). Access to the liquid chromatography-tandem mass spectrometry was made possible by the Operational Program Prague Compet- 
itiveness (OPPK) project (CZ.2.16/3.1.00/24012). This work has been generously supported by a research grant to J.P. Kraus from Orphan Technologies Ltd. and a gift of the Hempling Foundation for Homocystinuria Research.
Address correspondence to: Jan P. Kraus, University of Colorado, SOM, Department of Pediatrics, 12800 E. $19^{\text {th }}$ Ave, Mail Stop 8313, Aurora, Colorado 80045, USA. Phone: 303.724.3812; E-mail: jan.kraus@ucdenver.edu.
1. Carson NA, Neill DW. Metabolic abnormalities detected in a survey of mentally backward individuals in Northern Ireland. Arch Dis Child. 1962;37:505-513.

2. Mudd SH, Finkelstein JD, Irreverre F, Laster L. Homocystinuria: an enzymatic defect. Science. 1964;143(3613):1443-1445.

3. Miles EW, Kraus JP. Cystathionine $\beta$-synthase: structure, function, regulation, and location of homocystinuria-causing mutations. J Biol Chem. 2004;279(29):29871-29874.

4. Mudd SH, Levy HL, Kraus JP. Disorders of transsulfuration. In: Scriver CR, et al., eds. The Metabolic and Molecular Bases of Inherited Disease. New York, New York, USA: McGraw-Hill; 2001:2007-2056.

5. Kraus JP, Kožich V. Cystathionine- $\beta$-synthase and its deficiency. In: Carmel R, Jacobsen DW, eds. Homocysteine in Health and Disease. Cambridge, United Kingdom: Cambridge University Press; 2001:223-243.

6. Komrower GM, Lambert AM, Cusworth DC, Westall RG. Dietary treatment of homocystinuria. Arch Dis Child. 1966;41(220):666-671.

7. Barber GW, Spaeth GL. The successful treatment of homocystinuria with pyridoxine. JPediatr. 1969;75(3):463-478.

8 . Mudd SH, et al. The natural history of homocystinuria due to cystathionine $\beta$-synthase deficiency. Am J Hum Genet. 1985;37(1):1-31.

9. Picker JD, Levy HL. Homocystinuria caused by cystathionine $\beta$-synthase deficiency. In: Pagon RA, et al., ed. GeneReviews [Internet]. Seattle, Washington, USA: University of Washington, Seattle; 1993-2016.

10. Walter JH, Wraith JE, White FJ, Bridge C, Till J. Strategies for the treatment of cystathionine $\beta$-synthase deficiency: the experience of the Willink Biochemical Genetics Unit over the past 30 years. Eur J Pediatr. 1998;157(suppl 2):S71-S76.

11. Finkelstein JD. Methionine metabolism in mammals. J Nutr Biochem. 1990;1(5):228-237.

12. Huemer M, et al. Newborn screening for homocystinurias and methylation disorders: systematic review and proposed guidelines. JInherit Metab Dis. 2015;38(6):1007-1019.

13. Kraus JP, Rosenberg LE. Cystathionine beta-synthase from human liver: improved purification scheme and additional characterization of the enzyme in crude and pure form. Arch Biochem Biophys. 1983;222(1):44-52.

14. Ereno-Orbea J, Majtan T, Oyenarte I, Kraus JP, Martinez-Cruz LA. Structural insight into the molecular mechanism of allosteric activation of human cystathionine beta-synthase by S-adenosylmethionine. Proc Natl Acad Sci U S A.
2014;111(37):E3845-E3852.

15. Stabler SP, et al. Elevated plasma total homocysteine in severe methionine adenosyltransferase I/III deficiency. Metabolism. 2002;51(8):981-988.

16. Janosik M, Kery V, Gaustadnes M, Maclean KN, Kraus JP. Regulation of human cystathionine $\beta$-synthase by S-adenosyl-L-methionine: evidence for two catalytically active conformations involving an autoinhibitory domain in the C-terminal region. Biochemistry. 2001;40(35):10625-10633.

17. Frank N, Kery V, Maclean KN, Kraus JP. Solvent-accessible cysteines in human cystathionine beta-synthase: crucial role of cysteine 431 in S-adenosyl-L-methionine binding. Biochemistry. 2006;45(36):11021-11029.

18. Kery V, Poneleit L, Kraus JP. Trypsin cleavage of human cystathionine $\beta$-synthase into an evolutionarily conserved active core: structural and functional consequences. Arch Biochem Biophys. 1998;355(2):222-232.

19. Vugmeyster Y, Xu X, Theil FP, Khawli LA, Leach MW. Pharmacokinetics and toxicology of therapeutic proteins: Advances and challenges. World J Biol Chem. 2012;3(4):73-92.

20. Kang JS, Deluca PP, Lee KC. Emerging PEGylated drugs. Expert Opin Emerg Drugs. 2009;14(2):363-380.

21. Maclean KN, et al. A novel transgenic mouse model of CBS-deficient homocystinuria does not incur hepatic steatosis or fibrosis and exhibits a hypercoagulative phenotype that is ameliorated by betaine treatment. Mol Genet Metab. 2010;101(2-3):153-162.

22. Maclean $\mathrm{KN}$, et al. Cystathionine $\beta$-synthase null homocystinuric mice fail to exhibit altered hemostasis or lowering of plasma homocysteine in response to betaine treatment. Mol Genet Metab. 2010;101(2-3):163-171.

23. D'Souza A, Theis JD, Vrana JA, Dogan A. Pharmaceutical amyloidosis associated with subcutaneous insulin and enfuvirtide administration. Amyloid. 2014;21(2):71-75

24. Kitano H. Biological robustness. Nat Rev Genet. 2004;5(11):826-837.

25. Rothe M, Schambach A, Biasco L. Safety of gene therapy: new insights to a puzzling case. Curr Gene Ther. 2014;14(6):429-436.

26. Mudd SH, et al. Homocysteine and its disulfide derivatives: a suggested consensus terminology. Arterioscler Thromb Vasc Biol. 2000;20(7):1704-1706.

27. Mansoor MA, Svardal AM, Ueland PM. Determination of the in vivo redox status of cysteine, cysteinylglycine, homocysteine, and glutathione in human plasma. Anal Biochem. 1992;200(2):218-229.

28. Mansoor MA, Ueland PM, Aarsland A, Svardal
A. Redox status and protein binding of plasma homocysteine and other aminothiols in patients with homocystinuria. Metabolism. 1993;42(11):1481-1485.

29. Gupta S, et al. Mouse models of cystathionine beta-synthase deficiency reveal significant threshold effects of hyperhomocysteinemia. FASEB J. 2009;23(3):883-893.

30. Yap S, et al. Vascular outcome in patients with homocystinuria due to cystathionine beta-synthase deficiency treated chronically: a multicenter observational study. Arterioscler Thromb Vasc Biol. 2001;21(12):2080-2085.

31. Maclean KN, et al. Cystathionine protects against endoplasmic reticulum stress-induced lipid accumulation, tissue injury, and apoptotic cell death. J Biol Chem. 2012;287(38):31994-32005.

32. Volpe JJ, Laster L. Transsulfuration in fetal and postnatal mammalian liver and brain. Cystathionine synthase, its relation to hormonal influences, and cystathionine. Biol Neonate. 1972;20(5):385-403.

33. Dayal S, et al. Paradoxical absence of a prothrombotic phenotype in a mouse model of severe hyperhomocysteinemia. Blood. 2012;119(13):3176-3183.

34. Kobayashi S, et al. Cystathionine is a novel substrate of cystine/glutamate transporter: implications for immune function. J Biol Chem. 2015;290(14):8778-8788.

35. Gupta S, Melnyk SB, Kruger WD. Cystathionine $\beta$-synthase-deficient mice thrive on a low-methionine diet. FASEB J. 2014;28(2):781-790.

36. Watanabe M, et al. Mice deficient in cystathionine $\beta$-synthase: Animal models for mild and severe homocyst(e)inemia. Proc Natl Acad Sci US A. 1995;92(5):1585-1589.

37. Veronese FM, Pasut G. PEGylation, successful approach to drug delivery. Drug Discov Today. 2005;10(21):1451-1458.

38. Vellard M. The enzyme as drug: application of enzymes as pharmaceuticals. Curr Opin Biotechnol. 2003;14(4):444-450.

39. Kraus JP. Cystathionine $\beta$-synthase (human). Methods Enzymol. 1987;143:388-394.

40. Allen RH, Stabler SP, Lindenbaum J. Serum betaine, N,N-dimethylglycine and N-methylglycine levels in patients with cobalamin and folate deficiency and related inborn errors of metabolism. Metabolism. 1993;42(11):1448-1460.

41. Roman HB, Hirschberger LL, Krijt J, Valli A, Kozich V, Stipanuk MH. The cysteine dioxgenase knockout mouse: altered cysteine metabolism in nonhepatic tissues leads to excess H2S/HS(-) production and evidence of pancreatic and lung toxicity. Antioxid Redox Signal. 2013;19(12):1321-1336. 